\title{
Helicobacter pylori-induced modulation of the promoter methylation of Wnt antagonist genes in gastric carcinogenesis
}

\author{
Hyo-Joon Yang ${ }^{1} \cdot$ Sang Gyun Kim² ${ }^{2}$ Joo Hyun $\mathrm{Lim}^{3} \cdot \mathrm{Ji}$ Min Choi ${ }^{3} \cdot$ \\ Woo Ho Kim ${ }^{4}$ Hyun Chae Jung ${ }^{2}$
}

Received: 31 March 2017/ Accepted: 8 June 2017/Published online: 22 June 2017

(c) The International Gastric Cancer Association and The Japanese Gastric Cancer Association 2017

\begin{abstract}
Background This study aimed to investigate the changes in the promoter methylation and gene expression of multiple Wnt antagonists between the chronic infection and eradication of Helicobacter pylori (H. pylori) in gastric carcinogenesis.

Methods The levels of methylation and corresponding mRNA expression of seven Wnt antagonist genes (SFRP1, $-2,-5$, DKK1, -2, -3, WIF1) were compared among the patients with $H$. pylori-positive gastric cancers (GCs), and $H$. pylori-positive and $H$. pylori-negative controls, by quantitative MethyLight assay and real-time reverse transcription (RT)-polymerase chain reaction (PCR), respectively. The changes of the methylation and expression levels of the genes were also compared between the $H$.
\end{abstract}

Electronic supplementary material The online version of this article (doi:10.1007/s10120-017-0741-6) contains supplementary material, which is available to authorized users.

Sang Gyun Kim

harley1333@hanmail.net

1 Division of Gastroenterology, Department of Internal Medicine and Gastrointestinal Cancer Center, Kangbuk Samsung Hospital, Sungkyunkwan University School of Medicine, Seoul, Korea

2 Division of Gastroenterology, Department of Internal Medicine and Liver Research Institute, Seoul National University College of Medicine, 103 Daehak-ro, Jongno-gu, Seoul 03080, Korea

3 Department of Internal Medicine and Healthcare Research Institute, Healthcare System Gangnam Center, Seoul National University Hospital, Seoul, Korea

4 Department of Pathology, Seoul National University College of Medicine, Seoul, Korea pylori eradication and H. pylori-persistent groups 1 year after endoscopic resection of GCs.

Results The methylation levels of SFRP and DKK family genes were significantly increased in the patients with $H$. pylori-positive GCs and followed by $H$. pyloripositive controls compared with $H$. pylori-negative controls $(P<0.001)$. SFRP1, -2 , and DKK3 gene expression was stepwise downregulated from $H$. pylorinegative controls, $H$. pylori-positive controls, and to $H$. pylori-positive GCs $(P<0.05)$. Among the Wnt antagonists, only the degrees of methylation and downregulation of DKK3 were significantly reduced after $H$. pylori eradication $(P<0.05)$.

Conclusion Epigenetic silencing of SFRP and DKK family genes may facilitate the formation of an epigenetic field during $H$. pylori-associated gastric carcinogenesis. The epigenetic field may not be reversed even after $H$. pylori eradication except by DKK3 methylation.

Keywords Helicobacter pylori - Wnt signaling pathway · Intercellular signaling peptides and proteins - DNA methylation $\cdot$ Stomach neoplasms

\section{Introduction}

Worldwide, gastric cancer (GC) ranks fifth in incidence and third in mortality with 0.9 million cases and 0.7 million deaths estimated in 2012 [1]. In eastern Asia especially, half the world's cases occur and the morality rates are highest. In Korea and Japan, the majority of GC is detected early by nationwide cancer screening [2, 3], and endoscopic resection has become an effective alternative to surgery for indicated cases of early gastric cancers (EGCs) [4, 5]. However, the risk of development 
of metachronous GC in the remnant stomach after endoscopic resection is 6.7 times higher than in surgical resection [6], which suggests that the remnant gastric mucosae in patients with GC has already been affected by certain carcinogenic changes, forming a field of cancerization [7].

Aberrant DNA methylation is deeply involved in gastric carcinogenesis as a major mechanism of epigenetic alteration [8]. Inactivation of tumor suppressor genes by promoter hypermethylation was observed in GC [9], which was also induced by Helicobacter pylori (H. pylori) infection [10]. The levels of methylation were increased in noncancerous gastric mucosae of patients with GC and higher in cases with multiple GCs [11], which suggested that $H$. pylori infection could induce an epigenetic field for cancerization [12]. However, little has been known as to how the epigenetic field for GC is affected by the eradication of $H$. pylori $[13,14]$.

The Wnt/ $\beta$-catenin pathway has been known to have a crucial role in the development of GC [15, 16]. Activation of the Wnt/ $\beta$-catenin pathway is enhanced by $H$. pylori infection [17] and partly attenuated by $H$. pylori eradication [18]. Several studies have shown that the Wnt/ $\beta$-catenin pathway was activated by epigenetic inactivation of various Wnt antagonists such as the secreted frizzled-related protein (SFRP) family [19, 20], DICKKOPF (DKK) family [21], and Wnt inhibitory factor-1 (WIF1) [22] in human GCs. However, there has been no comprehensive study on promoter methylation and the subsequent downregulation of multiple Wnt antagonists in the noncancerous gastric mucosae of GC patients. Furthermore, whether the epigenetic field of Wnt antagonists is influenced by $H$. pylori infection and eradication has not been explored.

In this study, we aimed to evaluate whether epigenetic fields of Wnt antagonists are formed during gastric carcinogenesis and affected by $H$. pylori infection and eradication. First, we investigated the levels of promoter methylation and corresponding mRNA expression of seven Wnt antagonist genes (SFRP1, -2, 5, DKK1, -2, -3, and WIF1) in $H$. pylori-associated gastric carcinogenesis, represented as $H$. pylori-negative controls, H. pylori-positive controls, and $H$. pyloripositive patients with GCs. To assess the effect of $H$. pylori eradication as well as $H$. pylori infection, we modified the design by Maekita et al. [10] and focused on $H$. pylori-positive GCs. Then, to evaluate the effect of $H$. pylori eradication on the possible regression of the field, we compared changes in the gene methylation and mRNA expression levels between $H$. pylori eradication and persistence in patients 1 year after endoscopic resection of EGC.

\section{Methods}

\section{Subjects and tissue samples}

This study included 60 patients with $H$. pylori-positive GCs, $36 \mathrm{H}$. pylori-positive controls, and $36 \mathrm{H}$. pylori-negative controls. Among the patients with GCs, 30 patients were in the eradication group and the remaining 30 were the persistence group. Outpatients or screening recipients who underwent upper endoscopy and were diagnosed as normal or gastritis constituted the control group.

During endoscopy, two pieces of gastric mucosa were taken from the antrum and the corpus, respectively, for histological evaluation of inflammatory cell infiltration, atrophy, and intestinal metaplasia according to the updated Sydney scoring system [23]. The status of $H$. pylori was evaluated by both histology with modified Giemsa staining and a rapid urease test (CLO test; Delta West, Bently, Australia). H. pylori infection was considered as positive when at least one of the two results was positive [24].

Two biopsy samples for promoter methylation and mRNA expression analyses were obtained from antral mucosae of the control subjects. In the GC patients, two pieces of noncancerous antral mucosa were taken at baseline and after 1 year of endoscopic resection and subsequent $H$. pylori eradication or follow-up, respectively. This study was approved by the Institutional Review Board of Seoul National University Hospital (H-1405-003-574), and written informed consent was obtained from all subjects.

\section{DNA extraction, bisulfite modification, and MethyLight assay}

DNA was extracted from gastric mucosae and modified with sodium bisulfite as previously described [25]. In brief, specimens were dissolved in proteinase $\mathrm{K}$ solution $[20 \mathrm{mmol} / \mathrm{l}$ Tris-hydrochloride ( $\mathrm{pH} 8.0$ ), $10 \mathrm{mmol} / \mathrm{l}$ EDTA, $0.5 \%$ sodium dodecyl sulfate, and $20 \mathrm{mg} / \mathrm{ml}$ proteinase $\mathrm{K}$ ] followed by DNA isolation using phenol/chloroform extraction and ethanol precipitation. Genomic DNA of $1 \mu \mathrm{g}$ was bisulfite modified using the EZ DNA Methylation Kit (Zymo Research, Irvine, CA, USA) following the manufacturer's instructions.

The methylation status of seven Wnt antagonist genes from bisulfite-modified DNA samples was quantified using real-time polymerase chain reaction (PCR)-based MethyLight technology as previously reported [25-27]. MethyLight, as a sensitive, high-throughput methylation assay, provides highly specific detection of methylation by using probes that cover methylation sites as well as methylationspecific primers [27]. The primer and probe sequences used in the reaction are shown in Supplement Table S1 [28-31]. The quantified level of each gene was reported as a 
percentage of methylated reference (PMR), which is the relative methylation ratio of the target gene to the ALU gene of a sample divided by a ratio of the target gene to the ALU gene of sodium bisulfite and $\mathrm{CpG}$ methyltransferase (M.SssI)-treated sperm DNA and multiplied by 100 [27]. All samples were tested in triplicate.

\section{RNA extraction and quantitative reverse transcription (RT)-polymerase chain reaction (PCR)}

Gastric mucosae were homogenized followed by RNA isolation using Trizol Reagent (Invitrogen, Carlsbad, CA, USA) according to the manufacturer's instructions. Complementary DNA was synthesized from $1 \mu \mathrm{g}$ total RNA with M-MLV reverse transcription reagent (Invitrogen), and $4 \mu \mathrm{l}$ of resulting cDNA was amplified in $10 \mu \mathrm{l} 2 \mathrm{X}$ SYBR Green master mix (Takara Bio, Shiga, Japan) using ABI PRISM 7000 Sequence Detection System (Applied Biosystems, Foster City, CA, USA). The primer sequences and reaction conditions are presented in Supplement Table S2 [32-37]. Relative gene expression was determined by the $2^{-\Delta \Delta \mathrm{Ct}}$ method, and the human GAPDH gene was used as an endogenous reference [38]. All samples were tested in duplicate.

\section{Immunohistochemistry}

To evaluate the cytoplasmic and nuclear stain of $\beta$-catenin that suggests activation of the $\mathrm{Wnt} / \beta$-catenin pathway, immunohistochemistry (IHC) was performed. Because of the limited availability of formalin-fixed, paraffin-embedded tissues, three cases from each group were stained with a monoclonal antibody for $\beta$-catenin (BD Biosciences, San Diego, CA, USA; dilution 1:800) using Ventana BenchMark XT staining systems (Ventana Medical Systems, Tucson, AZ, USA) as previously described [39].

The expression of $\beta$-catenin was evaluated semiquantitatively based on previous reports $[17,18,40]$. For evaluation of the membranous stain, $H$. pylori-negative controls were set as reference. Membranous expression of $\beta$-catenin in the H. pylori-positive controls or GCs was considered as 'maintained' when both the intensity and the frequency were similar to those in the $H$. pylori-negative controls, 'reduced' when the staining was decreased, and 'absent' when membranous staining are not observed. In the evaluation of cytoplasmic and nuclear stain, low- and highlevel expression was defined as $<10 \%$ and $>10 \%$ of cells with positive cytoplasmic or nuclear $\beta$-catenin staining.

\section{Statistical analysis}

For comparison between GC and controls, the KruskalWallis or Mann-Whitney $U$ test for continuous variables, and Pearson's chi-squared test or Fisher's exact test for the categorical variables, were used. The gene methylation or expression levels at baseline and at 1-year follow-up were compared using the Wilcoxon signed-rank test for continuous variables and the McNemar test for categorical variables. All statistical analyses were performed with SPSS (version 21.0; SPSS, Chicago, IL, USA). $P<0.05$ was considered significant.

\section{Results}

\section{Subject characteristics}

Clinicopathological characteristics of the study subjects are summarized in Table 1. There were significant differences in demographics among the $H$. pylori-negative and $H$. pylori-positive controls and the patients with $H$. pyloripositive GCs. The patients with H. pylori-positive GCs were oldest [median, 60.2 years; interquartile range (IQR), 53.5-68.1] and most likely to be male $(65 \%, 39 / 60)$, followed by $H$. pylori-positive controls and then $H$. pylorinegative controls (both $P<0.001$ ). Histological characteristics were also different among the groups. The infiltration of neutrophils and monocytes was more prominent in the gastric mucosae of the patients with $H$. pylori-positive GCs and controls than in those of $H$. pylori-negative controls (both $P<0.001$ ). In addition, intestinal metaplasia was more severe in the patients with GCs than in the controls $(P<0.001)$.

\section{Aberrant DNA methylation and reduced mRNA expression of Wnt antagonists in $\mathrm{H}$. pylori-associated gastric carcinogenesis}

The methylation levels of seven Wnt antagonist genes in gastric samples of the three groups were quantified. In the MethyLight assay, methylation levels of SFRP and DKK family genes in the gastric mucosae were very low in $H$. pylori-negative controls with median PMR values less than 5 (Fig. 1a-f; Supplement Table S3). They were increased in $H$. pylori-positive controls, with median PMR values up to 12.4 and became highest in the noncancerous mucosae of $H$. pylori-positive GCs, although the methylation levels were quite variable among the cases, and genes with median PMR values ranged between 8.2 and 34.1. The stepwise increment in the methylation levels of the genes from $H$. pylori-negative controls, H. pylori-positive controls, and H. pylori-positive GCs were statistically significant (all $P<0.001$ ). Although the methylation level of WIF1 was increased with $H$. pylori infection, it was rather decreased to a lower level in patients with GCs (Fig. 1g). 
Table 1 Clinicopathological characteristics of study subjects

\begin{tabular}{|c|c|c|c|c|}
\hline & $H p$-negative controls $(n=36)$ & $H p$-positive controls $(n=36)$ & $H p$-positive GCs $(n=60)$ & $P$ value \\
\hline Age, median (IQR), years & $45.5(31.8-61.5)$ & $49.5(40.0-57.8)$ & $60.2(53.5-68.1)$ & $<0.001$ \\
\hline Sex, $n(\%)$ & & & & $<0.001$ \\
\hline Female & $27(75.0)$ & $25(69.4)$ & $21(35.0)$ & \\
\hline Male & $9(25.0)$ & $11(30.6)$ & $39(65.0)$ & \\
\hline Neutrophils, $n(\%)$ & & & & $<0.001$ \\
\hline None to mild & $33(91.7)$ & $3(8.3)$ & $2(3.3)$ & \\
\hline Moderate to severe & $3(8.3)$ & $33(91.7)$ & $58(96.7)$ & \\
\hline Monocyte, $n(\%)$ & & & & $<0.001$ \\
\hline None to mild & $28(72.2)$ & $1(2.8)$ & $6(10.0)$ & \\
\hline Moderate to severe & $10(27.8)$ & $35(97.2)$ & $54(90.0)$ & \\
\hline Atrophy, $n(\%)$ & & & & 0.135 \\
\hline None to mild & $20 / 22(90.0)$ & 22/24 (91.7) & $37 / 47(78.7)$ & \\
\hline Moderate to severe & $2 / 22(9.1)$ & $2 / 24(8.3)$ & $10 / 47(21.3)$ & \\
\hline Intestinal metaplasia, $n(\%)$ & & & & $<0.001$ \\
\hline None to mild & $36(100)$ & $36(100)$ & $28(46.7)$ & \\
\hline Moderate to severe & $0(0)$ & $0(0)$ & $32(53.3)$ & \\
\hline
\end{tabular}

Hp Helicobacter (H.) pylori; GC gastric cancer; $I Q R$ interquartile range

Next, mRNA expression levels of Wnt antagonists were compared among the three groups. In real-time RT-PCR, levels of mRNA expression of SFRP and DKK family genes were downregulated in $H$. pylori-positive controls compared to $H$. pylori-negative controls and decreased further in the noncancerous mucosae of patients with $H$. pylori-positive GCs (Fig. 1a-f). In particular, reduction of gene expression was statistically significant in SFRP1, -2, and DKK3 genes (all $P<0.05$ ).

In IHC staining for $\beta$-catenin, all three cases in the $H$. pylori-negative controls showed almost exclusively membranous stain and negativity for cytoplasmic or nuclear stain (Fig. 2a). All three cases of $H$. pylori-positive controls showed low-level expression of cytoplasmic and nuclear $\beta$-catenin, although membranous staining was maintained (Fig. 2b). In three H. pylori-positive patients with GC, two cases showed high-level expression and the one remaining case showed low-level expression of cytoplasmic and nuclear $\beta$-catenin, but membranous staining was also maintained in all cases compared to $H$. pylorinegative controls (Fig. 2c, d).

\section{Effect of $\boldsymbol{H}$. pylori eradication on DNA methylation and mRNA expression of Wnt antagonists}

The patients with $H$. pylori-positive GC were divided into eradication and persistence groups. Although baseline characteristics were not different between the two groups (all $P>0.05$ ), the difference in the pathological characteristics in the gastric mucosae became prominent at 1-year follow-up (Table 2). Neutrophil and monocyte infiltration was less prominent (both $P<0.001$ ), and atrophy was less severe $(P=0.045)$ in the eradication group than in the persistent group, although severity of intestinal metaplasia was similar between the groups.

Methylation levels of Wnt antagonists were not reduced at 1 year after $H$. pylori eradication compared to the baseline value (Fig. 3a-e, g), except for the methylation level of DKK3, which was significantly decreased in the eradication group $(P=0.017)$ although it was not in the persistent group (Fig. 3f). Rather, there were tendencies of increasing levels of DNA methylation of SFRP1, -2, -5 and DKK1 and -2 genes irrespective of $H$. pylori eradication (Supplement Table S3). The levels of mRNA expression of these genes were significantly reduced in both the eradication and persistent groups (Fig. 3a). However, the expression of DKK3 at 1 year after $H$. pylori eradication was similar to the baseline value and significantly less reduced in the eradication group than in the persistent group $(P=0.023)$ (Fig. 3f).

There were no apparent changes in the expression of cytoplasmic and nuclear $\beta$-catenin between baseline and follow-up in both the eradication group (Fig. 2c, e) and the persistent group (Fig. 2d, f).

\section{Discussion}

In the present study, we evaluated the formation of an epigenetic field for cancerization of Wnt antagonists during H. pylori-associated gastric carcinogenesis. The MethyLight assay, quantitative methylation-specific PCR, 
Fig. 1 DNA methylation levels and corresponding mRNA expression levels in the seven Wnt antagonist genes: SFRP1 (a), SFRP2 (b), SFRP5 (c), $D K K 1(\mathbf{d}), D K K 2(\mathbf{e}), D K K 3(\mathbf{f})$, and $W I F 1$ (g) among Helicobacter pylori-negative controls, $H$. pylori-positive controls, and $H$. pylori-positive patients with gastric cancers. Results of DNA methylation are given as medians and interquartile ranges. Results of mRNA expression are presented as fold change compared to $H$. pylori-negative controls and standard deviations. With $H$. pylori-associated gastric carcinogenesis, methylation levels in SFRP1, -2, -5 and DKK1, -2 , and -3 were significantly increased, and mRNA expression levels in SFRP 1, SFRP 2, and DKK3 were significantly reduced. $P M R$ percentage of methylated reference; Hp Helicobacter ( $H$.) pylori; $G C$ gastric cancer

\section{SFRP1}
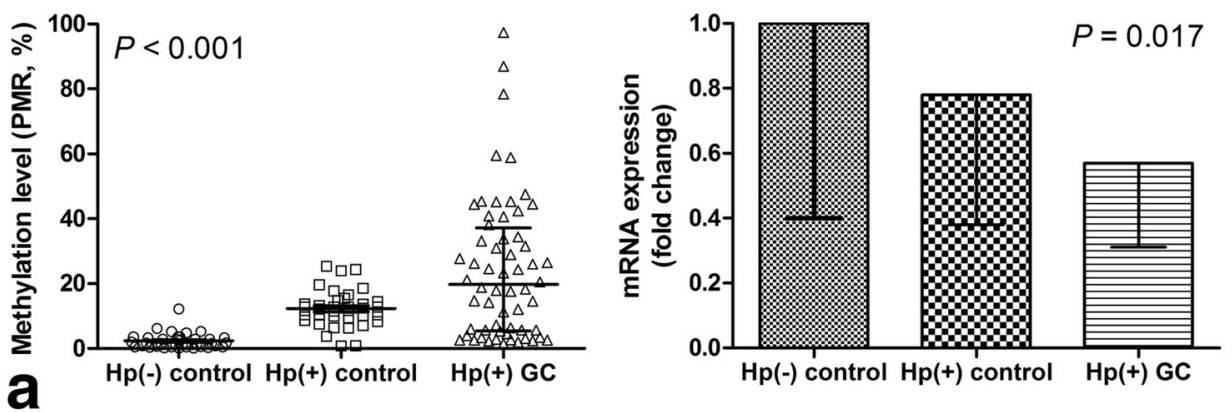

\section{SFRP2}
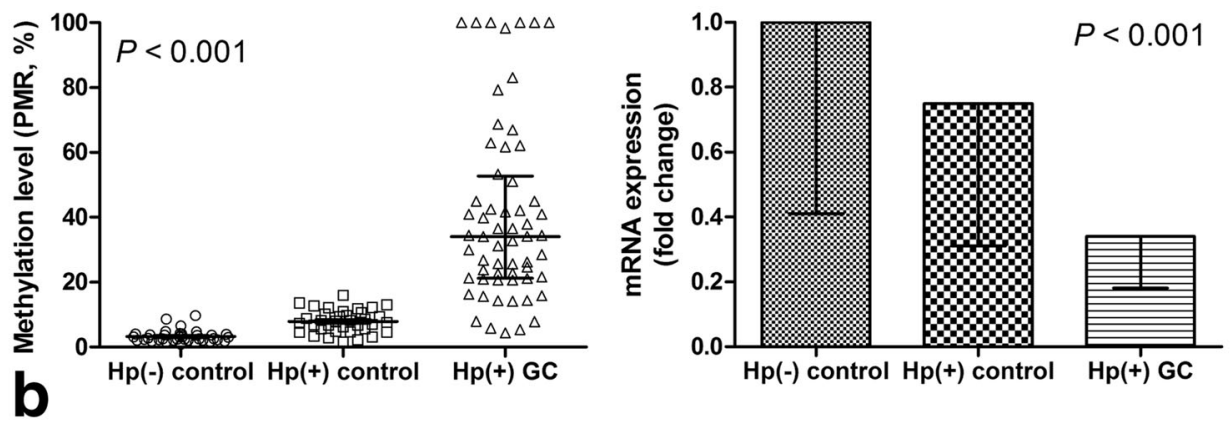

\section{SFRP5}
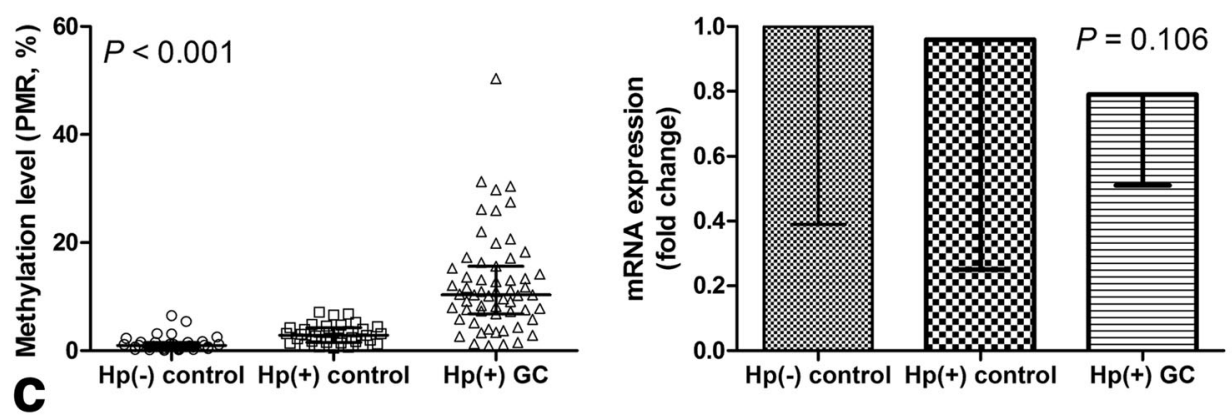

\section{DKK1}
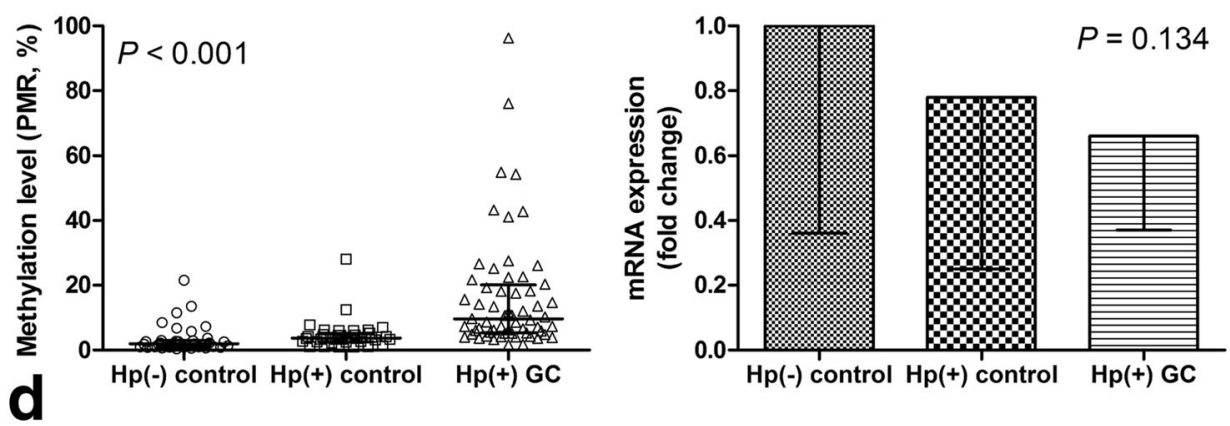
Fig. 1 continued
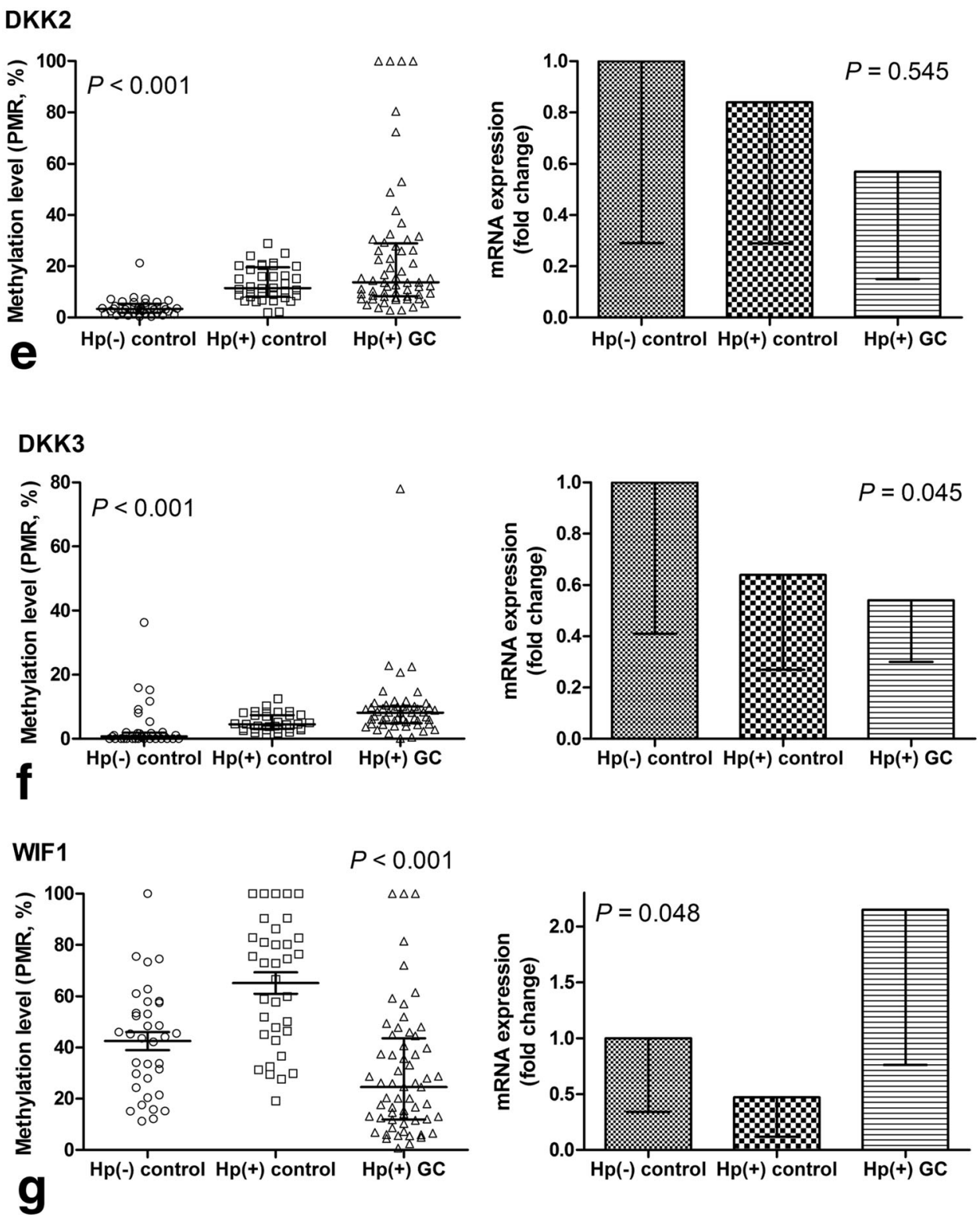

revealed that methylation levels of SFRP and DKK family gene promoters were increased in the noncancerous mucosae of the patients with $H$. pylori-positive GCs, which were higher than $H$. pylori-positive controls and even higher than $H$. pylori-negative controls. Real-time RT-PCR showed corresponding stepwise downregulation of SFRP1, -2, and DKK3 gene expression from $H$. pylori-negative controls to $H$. pylori-positive controls and then to $H$. pylori-positive GCs. The effects of $H$. pylori eradication on the field were also explored. Among the Wnt antagonists, the degree of methylation and downregulation were significantly reduced only in DKK3.

The epigenetic field in gastric carcinogenesis is becoming an important issue as endoscopic resection has become widely performed for the treatment of EGC $[4,5]$.
This stage has occurred because not only is the risk of metachronous recurrence highly increased after endoscopic resection [6], but such recurrence also has an important effect on the prognosis of the patients [41]. Epigenetic alteration has been suggested as a possible mechanism for field cancerization. Previous studies showed that methylation-dependent silencing of tumor suppressor genes such as p16, CDH1, and THBD was present and associated with $H$. pylori infection in noncancerous gastric mucosae of GC patients [8, 12, 42]. Recently, a relationship has emerged on the Wnt/ $\beta$-catenin and $H$. pylori infection in gastric carcinogenesis [43]. The $\mathrm{Wnt} / \beta$-catenin pathway was also implicated in GC by epigenetic silencing of multiple Wnt antagonists [19-22]. However, the formation of an epigenetic field of Wnt antagonists in $H$. pylori infection and 

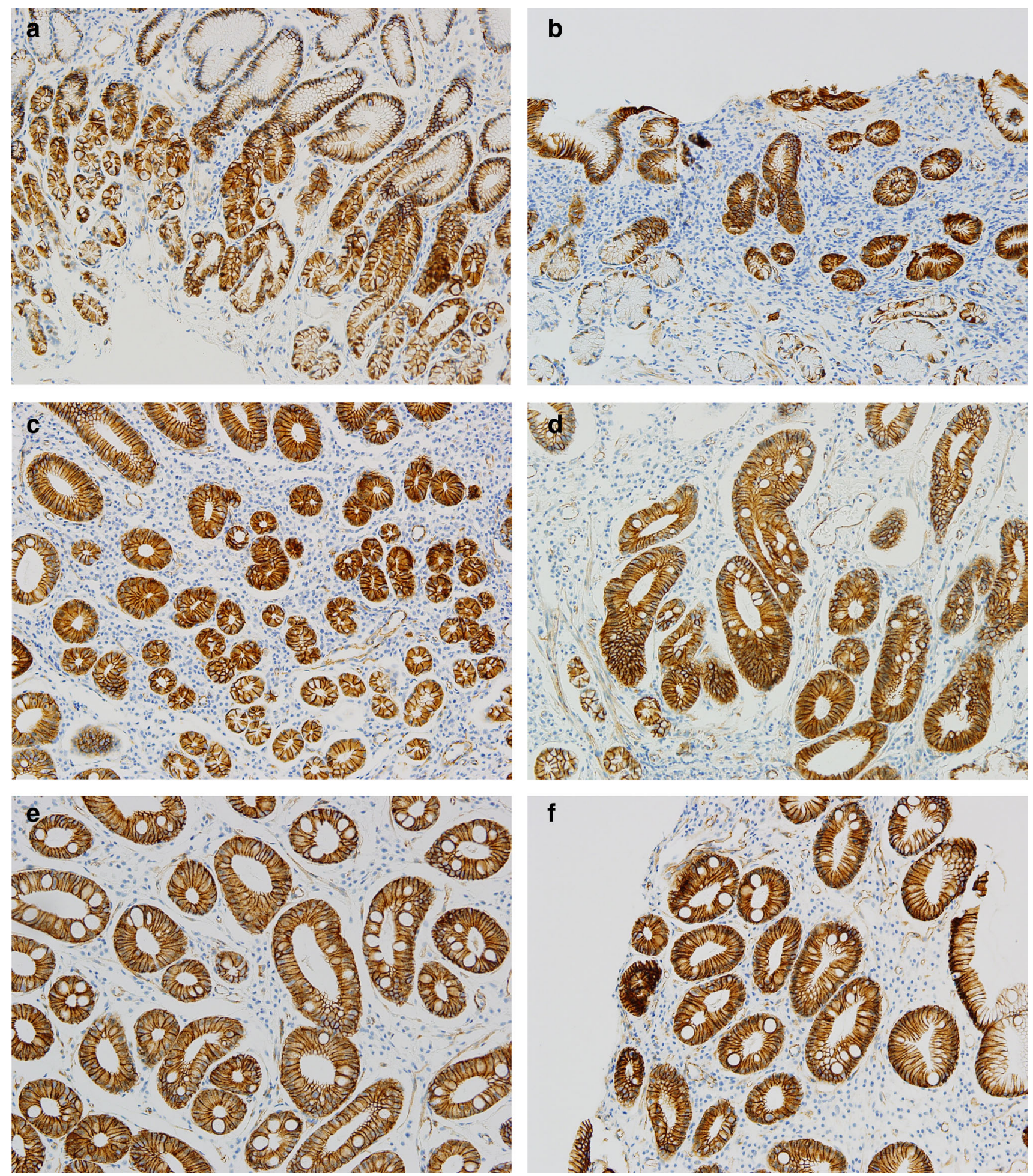

Fig. 2 Representative immunohistochemical staining for $\beta$-catenin. $\times 200$. a Gastric mucosae of $H$. pylori-negative control showed continuous membranous staining without cytoplasmic or nuclear expression of $\beta$-catenin. b Gastric mucosae of $H$. pylori-positive control demonstrated both maintained membranous staining and lowlevel expression of cytoplasmic and nuclear stating of $\beta$-catenin. $\mathbf{c}$, d Noncancerous mucosa of $H$. pylori-positive patients with gastric

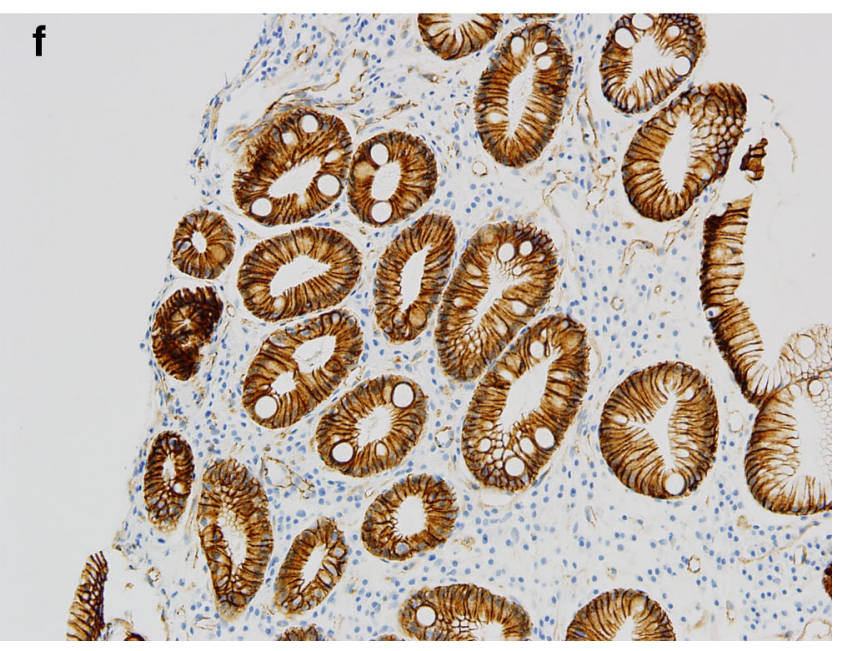

cancers revealed high-level expression of cytoplasmic and nuclear $\beta$ catenin as well as maintained membranous staining. e One-year follow-up sample of the case $\mathbf{c}$ after $H$. pylori eradication. $\mathbf{f}$ One-year follow-up sample of the case $\mathbf{d}$ with persistent $H$. pylori infection. There were no apparent changes in cytoplasmic and nuclear $\beta$-catenin staining and membranous staining between baseline and follow-up irrespective of $H$. pylori eradication 
Table 2 Clinicopathological difference between Helicobacter pylori eradication and persistent groups

\begin{tabular}{|c|c|c|c|c|c|}
\hline & \multicolumn{2}{|c|}{$H p$ eradication group $(n=30)$} & \multicolumn{2}{|c|}{$H p$ persistent group $(n=30)$} & \multirow[t]{2}{*}{$P$ value* } \\
\hline & Baseline & 1-year follow-up & Baseline & 1-year follow-up & \\
\hline Age, median (IQR), years & $61.7(51.7-68.4)$ & & $59.5(54.7-66.1)$ & & \\
\hline \multicolumn{6}{|l|}{ Sex, $n(\%)$} \\
\hline Female & $9(30.0)$ & & $12(40.0)$ & & \\
\hline Male & $21(70.0)$ & & $18(60.0)$ & & \\
\hline Neutrophils, $n(\%)$ & & & & & $<0.001$ \\
\hline None to mild & $1(3.3)$ & $30(100.0)$ & $1(3.3)$ & $0(0.0)$ & \\
\hline Moderate to severe & $29(96.7)$ & $0(0.0)$ & $29(96.7)$ & $30(100.0)$ & \\
\hline Monocytes, $n(\%)$ & & & & & $<0.001$ \\
\hline None to mild & $3(10.0)$ & $14(46.7)$ & $3(10.0)$ & $0(0.0)$ & \\
\hline Moderate to severe & $27(90.0)$ & $16(53.3)$ & $27(90.0)$ & $30(100.0)$ & \\
\hline Atrophy, $n(\%)$ & & & & & 0.045 \\
\hline None to mild & 19/24 (79.2) & $18 / 25(72.0)$ & $18 / 23(78.3)$ & $10 / 23(43.5)$ & \\
\hline Moderate to severe & $5 / 24(20.8)$ & $7 / 25(28.0)$ & $5 / 23(21.7)$ & $13 / 23(56.5)$ & \\
\hline Intestinal metaplasia, $n(\%)$ & & & & & 0.184 \\
\hline None to mild & $13(43.3)$ & $14(46.7)$ & $15(50.0)$ & $9(30.0)$ & \\
\hline Moderate to severe & $17(56.7)$ & $16(53.3)$ & $15(50.0)$ & $21(70.0)$ & \\
\hline
\end{tabular}

Hp H. pylori, IQR interquartile range

* One-year follow-up results between $H$. pylori eradication and persistent groups were compared

gastric carcinogenesis and the effect of $H$. pylori eradication on the field have not been investigated. Moreover, most previous studies were limited in that they utilized a nonquantitative method to detect promoter methylation.

In our study, H. pylori infection was associated with promoter methylation and downregulation of all Wnt antagonist genes. IHC staining showed increased expression of cytoplasmic and nuclear $\beta$-catenin. These findings are consistent with a previous study [17] that showed $H$. pylori infection led to $\beta$-catenin nuclear accumulation, and we additionally found that $H$. pylori-induced dysregulation of $\beta$-catenin might be mediated by methylation-dependent silencing of Wnt antagonists. We also showed that the levels of promoter methylation of the SFRP and DKK family genes was higher and their mRNA expression was lower in the noncancerous mucosae of $H$. pylori-positive GC patients than in $H$. pylori-positive controls. These findings corroborate previous studies of SFRP and DKK family genes on GC cell lines and tissues [19-21]. Our study further demonstrated that these epigenetic inactivations were also observed in noncancerous gastric mucosae of GC cases, which is also supported by IHC staining that demonstrated more prominent and frequent nuclear $\beta$ catenin staining in GC cases. This finding may explain the observation in IHC staining that demonstrated low- to high-level cytoplasmic and nuclear $\beta$-catenin staining in the noncancerous mucosa of $H$. pylori-positive patients with GCs, although the number of samples was too small and the expression was evaluated semiquantitatively.
Taken together, our results suggest that SFRP and DKK family genes may be involved in the formation of an epigenetic field in $H$. pylori-associated gastric carcinogenesis.

However, there are two points that should be considered in the interpretation of the aberrant DNA methylation of SFRP and DKK family genes in noncancerous mucosae of H. pylori-positive GC. First, methylation levels of those genes were highly variable. Similar findings were observed in a previous study [10]. This variation seems to occur because aberrant methylation develops in only a fraction of cells, as suggested. Second, DNA methylation is closely associated with the presence of intestinal metaplasia of gastric mucosae. It was reported that hypermethylation of THBD, HAND1, and APC was associated with intestinal metaplasia [42]. In our study, intestinal metaplasia was more severe in the mucosae of GC patients than in the controls. Thus, the increased level of methylation in Wnt antagonist genes may also be associated with the severity of metaplasia. Further study is required whether DNA methylation of Wnt antagonist genes is associated with intestinal metaplasia in patients without GCs.

It was noteworthy that methylation levels of WIF1 in patients with GCs were lower than those in $H$. pyloripositive and $H$. pylori-negative controls, whereas they were higher in the $H$. pylori-positive controls then in the $H$. pylori-negative controls. In a prior study, frequent hypermethylation and downregulation of WIF1 were observed in GC cell lines and tissues [22]. This finding may be interpreted that epigenetic silencing of WIF1 may emerge after 
Fig. 3 DNA methylation levels and corresponding mRNA expression levels in the seven Wnt antagonist genes: SFRP1 (a), SFRP2 (b), SFRP5 (c), $D K K 1$ (d), DKK2 (e), DKK3 (f), and WIF1 (g) in H. pyloripositive patients with gastric cancers at baseline and 1-year follow-up in whom $H$. pylori was eradicated ( $H p$ eradication) or not ( $H p$ persistent). After $H$. pylori eradication, methylation level only in DKK3 was significantly reduced $(P=0.017)$. Accordingly, mRNA expression level of DKK3 was significantly less reduced in the eradicated group ( $H p$ eradication) than in the persistent group ( $H p$ persistent) $(P=0.023) . P M R$ percentage of methylated reference, $\mathrm{Hp} \mathrm{H}$. pylori
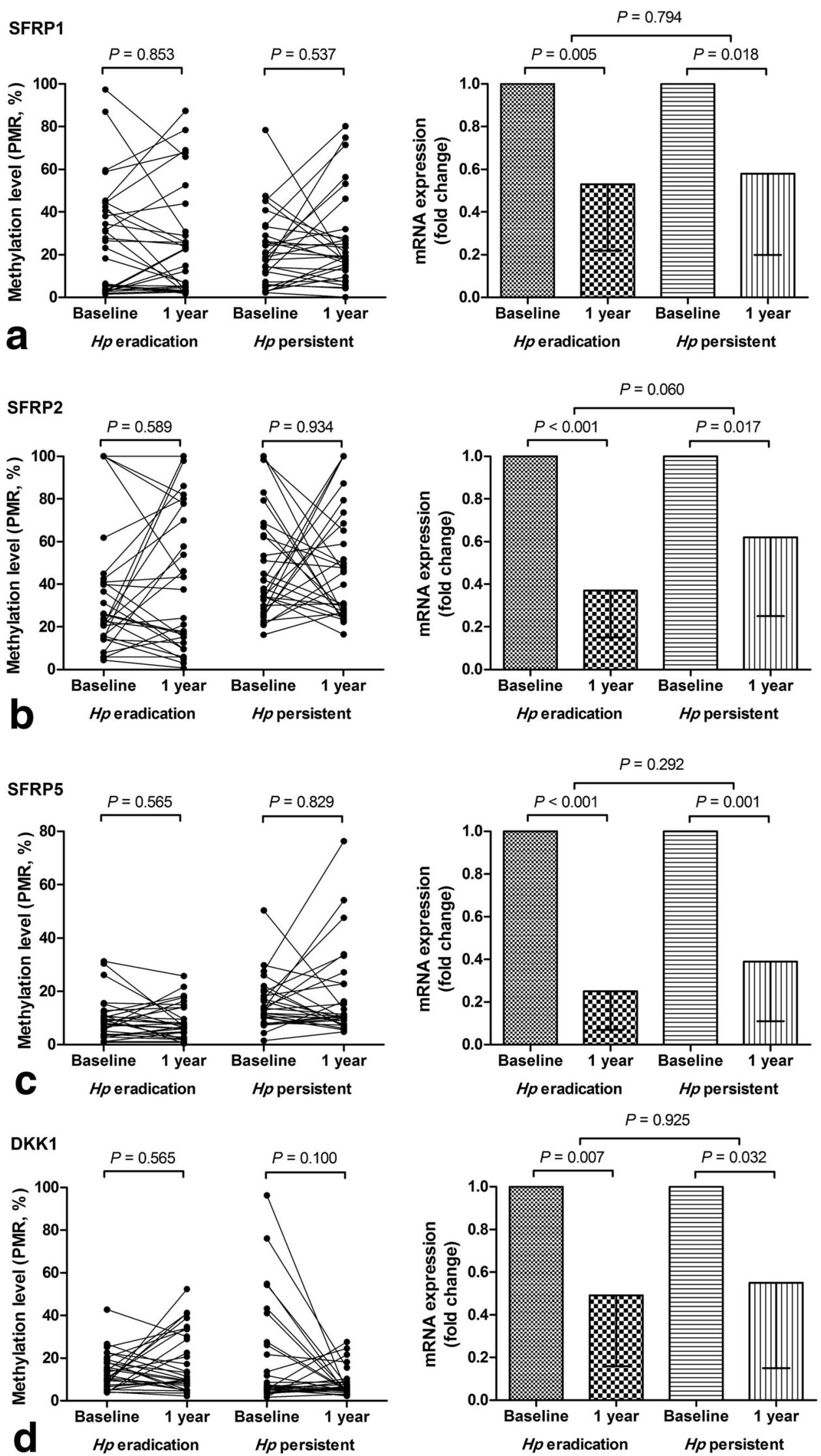
Fig. 3 continued
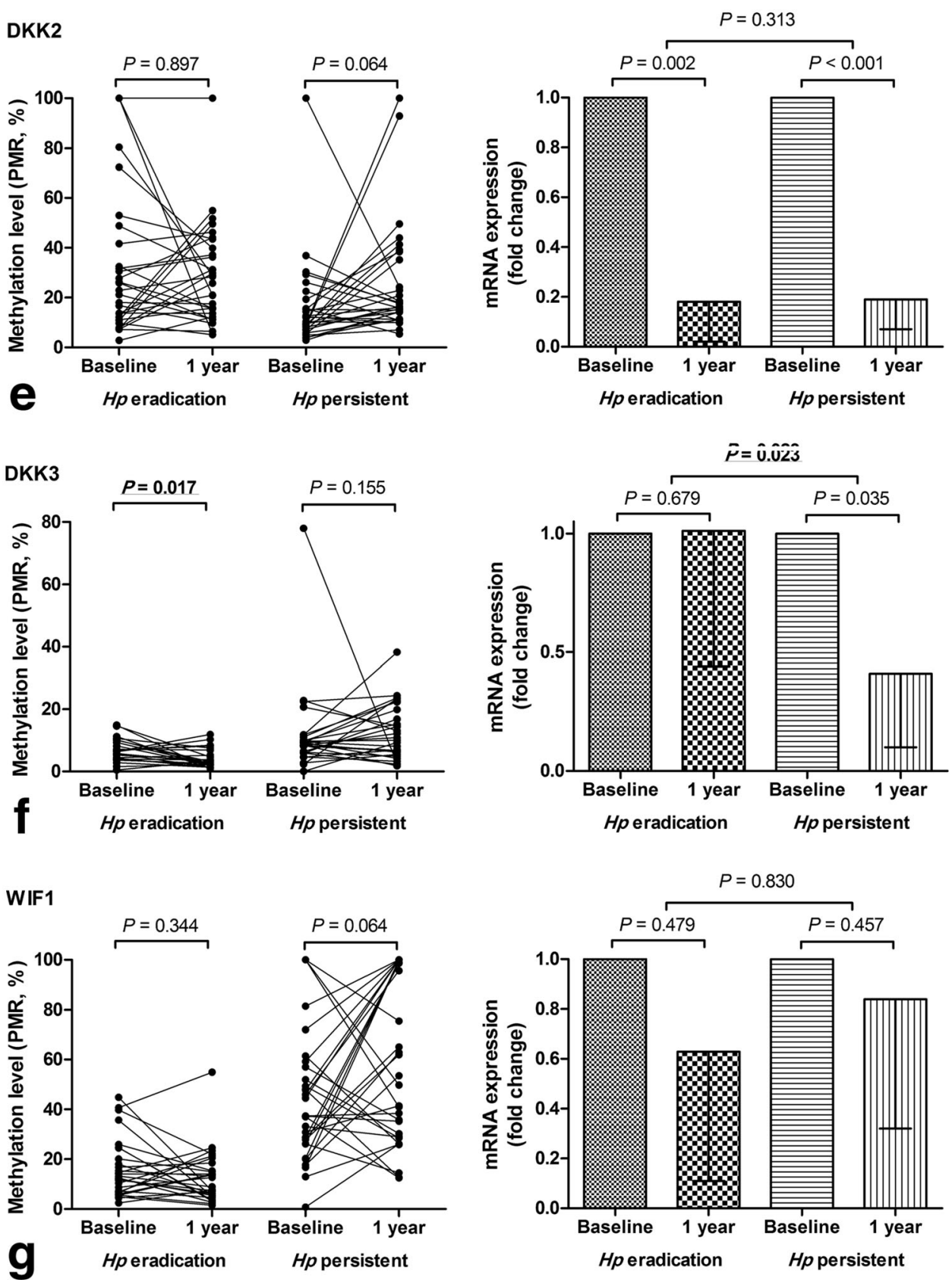

the development of GC rather than before cancer development or during the formation of epigenetic field.

Few studies conducted controlled comparison between eradication and persistent groups in the evaluation of the effect of $\mathrm{H}$. pylori eradication on promoter hypermethylation as we performed in the present study [25]. In our study, increased methylation levels of DKK3 were decreased, although those of SFPR and other DKK family genes were not, at 1 year after $H$. pylori eradication. This finding is consistent with a prior longitudinal study that showed that $H$. pylori eradication reduced methylation levels in LOX but not in APC [14], suggesting that the effect of $H$. pylori eradication on the reduction of aberrant
DNA methylation may be gene specific. It is interesting that mRNA levels of SFRP1, $-2,-3$ and DKK1 and -2 were significantly downregulated at 1-year follow-up from the baseline in both eradication and persistent groups. These changes may be explained by the tendencies of increasing methylation levels of these genes observed in both the eradication and persistent groups, although the extent of increased methylation levels was not significantly high (Supplement Table S4). Another possible explanation could be that an epigenetic mechanism other than DNA methylation, such as histone modification, might have also involved in the reduced mRNA expression [44]. Nevertheless, these results may suggest that $H$. pylori eradication 
could not lead to reduced DNA methylation and increased mRNA expression in those genes, at least in 1 year of follow-up. Because aberrant cytoplasmic and nuclear $\beta$ catenin was not decreased, it may be suggested that, once the epigenetic field of Wnt antagonists had been generated, it may persist even after $H$. pylori eradication, at least for 1 year. In the follow-up sample, mucosal atrophy and inflammatory cell infiltration were more severe in the persistent group than in the eradication group. Ongoing inflammation induced by persistent $H$. pylori infection could have resulted in aggravated mucosal atrophy. Thus, further study may be warranted to elucidate whether these differences may lead to a difference in the DNA methylation levels of SFRP and DKK family genes other than DKK3 in the longer-term follow-up.

The strengths of this study include adoption of quantitative methods in the evaluation of DNA methylation and mRNA expression and controlled design comparing $H$. pylori eradication with persistent groups. However, there are also some limitations. First, we did not include patients with $H$. pylori-negative GC. It is known that noncancerous gastric mucosae of $H$. pylori-negative patients with GC are at higher risk of developing metachronous cancer than those of $H$. pylori-positive patients [45]. Although our study has an advantage in involving a $H$. pylori eradication group, the presence of $H$. pylori-negative patients with GC could have provided additional insights in gastric carcinogenesis as in the previous studies [10, 42]. Second, IHC staining was conducted on only three cases and assessed semiquantitatively. For more exact evaluation, quantitative assessment using the ratio of $\beta$-catenin expression for the entire cohort of cases should have been done. In addition, immunofluorescence is necessary to evaluate accurately the changes in $\beta$-catenin expression patterns. Thus, caution is required when interpreting the results of IHC of the present study.

In conclusion, epigenetic silencing of SFRP and DKK family genes may mediate the formation of the epigenetic field during $H$. pylori-associated gastric carcinogenesis. Although most of these changes may persistent even after $H$. pylori eradication once the field has been developed, methylation of DKK3 may be reversed by $H$. pylori eradication in GC patients.

Acknowledgements This work was supported by a Grant from the Promising-Pioneering Researcher Program through Seoul National University in 2015 and by Korean College of Helicobacter and Upper Gastrointestinal Research.

\section{Compliance with ethical standards}

Conflict of interest The authors declare that they have no conflict of interests.
Human rights statement and informed consent This study was approved by Institutional Review Boards of Seoul National University Hospital (H-1405-003-574), and written informed consent was obtained from all subjects. All procedures followed were in accordance with the ethical standards of the responsible committee on human experimentation (institutional and national) and with the Helsinki Declaration of 1964 and later versions.

\section{References}

1. Ferlay J, Soerjomataram I, Dikshit R, Eser S, Mathers C, Rebelo $\mathrm{M}$, et al. Cancer incidence and mortality worldwide: sources, methods and major patterns in GLOBOCAN 2012. Int J Cancer. 2015;136:E359-86.

2. Chung SJ, Park MJ, Kang SJ, Kang HY, Chung GE, Kim SG, et al. Effect of annual endoscopic screening on clinicopathologic characteristics and treatment modality of gastric cancer in a highincidence region of Korea. Int J Cancer. 2012;131:2376-84.

3. Hosokawa O, Miyanaga T, Kaizaki Y, Hattori M, Dohden K, Ohta K, et al. Decreased death from gastric cancer by endoscopic screening: association with a population-based cancer registry. Scand J Gastroenterol. 2008;43:1112-5.

4. Pyo JH, Lee H, Min BH, Lee JH, Choi MG, Lee JH, et al. Longterm outcome of endoscopic resection vs. surgery for early gastric cancer: a non-inferiority-matched cohort study. Am J Gastroenterol. 2016;111:240-9.

5. Isomoto H, Shikuwa S, Yamaguchi N, Fukuda E, Ikeda K, Nishiyama $\mathrm{H}$, et al. Endoscopic submucosal dissection for early gastric cancer: a large-scale feasibility study. Gut. 2009;58:331-6.

6. Choi KS, Jung HY, Choi KD, Lee GH, Song HJ, Kim do H, et al. EMR versus gastrectomy for intramucosal gastric cancer: comparison of long-term outcomes. Gastrointest Endosc. 2011;73:942-8.

7. Ando T, Yoshida T, Enomoto S, Asada K, Tatematsu M, Ichinose $\mathrm{M}$, et al. DNA methylation of microRNA genes in gastric mucosae of gastric cancer patients: its possible involvement in the formation of epigenetic field defect. Int $\mathrm{J}$ Cancer. 2009; 124:2367-74.

8. Ushijima T, Hattori N. Molecular pathways: involvement of Helicobacter pylori-triggered inflammation in the formation of an epigenetic field defect, and its usefulness as cancer risk and exposure markers. Clin Cancer Res. 2012;18:923-9.

9. Ushijima T, Sasako M. Focus on gastric cancer. Cancer Cell. 2004;5:121-5.

10. Maekita T, Nakazawa K, Mihara M, Nakajima T, Yanaoka K, Iguchi $\mathrm{M}$, et al. High levels of aberrant DNA methylation in Helicobacter pylori-infected gastric mucosae and its possible association with gastric cancer risk. Clin Cancer Res. 2006;12:989-95.

11. Nakajima T, Maekita T, Oda I, Gotoda T, Yamamoto S, Umemura $\mathrm{S}$, et al. Higher methylation levels in gastric mucosae significantly correlate with higher risk of gastric cancers. Cancer Epidemiol Biomarkers Prev. 2006;15:2317-21.

12. Ushijima T. Epigenetic field for cancerization. J Biochem Mol Biol. 2007;40:142-50.

13. Nakajima T, Enomoto S, Yamashita S, Ando T, Nakanishi Y, Nakazawa K, et al. Persistence of a component of DNA methylation in gastric mucosae after Helicobacter pylori eradication. J Gastroenterol. 2010;45:37-44.

14. Shin CM, Kim N, Lee HS, Park JH, Ahn S, Kang GH, et al. Changes in aberrant DNA methylation after Helicobacter pylori 
eradication: a long-term follow-up study. Int $\mathrm{J}$ Cancer. 2013;133:2034-42.

15. Oshima H, Matsunaga A, Fujimura T, Tsukamoto T, Taketo MM, Oshima M. Carcinogenesis in mouse stomach by simultaneous activation of the Wnt signaling and prostaglandin E2 pathway. Gastroenterology. 2006;131:1086-95.

16. Woo DK, Kim HS, Lee HS, Kang YH, Yang HK, Kim WH. Altered expression and mutation of beta-catenin gene in gastric carcinomas and cell lines. Int J Cancer. 2001;95:108-13.

17. Franco AT, Israel DA, Washington MK, Krishna U, Fox JG, Rogers $\mathrm{AB}$, et al. Activation of beta-catenin by carcinogenic Helicobacter pylori. Proc Natl Acad Sci USA. 2005; 102:10646-51.

18. Hung KH, Wu JJ, Yang HB, Su LJ, Sheu BS. Host Wnt/betacatenin pathway triggered by Helicobacter pylori correlates with regression of gastric intestinal metaplasia after $H$. pylori eradication. J Med Microbiol. 2009;58:567-76.

19. Nojima M, Suzuki H, Toyota M, Watanabe Y, Maruyama R, Sasaki S, et al. Frequent epigenetic inactivation of SFRP genes and constitutive activation of Wnt signaling in gastric cancer. Oncogene. 2007;26:4699-713.

20. Cheng YY, Yu J, Wong YP, Man EP, To KF, Jin VX, et al. Frequent epigenetic inactivation of secreted frizzled-related protein 2 (SFRP2) by promoter methylation in human gastric cancer. Br J Cancer. 2007;97:895-901.

21. Sato H, Suzuki H, Toyota M, Nojima M, Maruyama R, Sasaki S, et al. Frequent epigenetic inactivation of DICKKOPF family genes in human gastrointestinal tumors. Carcinogenesis (Oxf). 2007;28:2459-66.

22. Taniguchi H, Yamamoto H, Hirata T, Miyamoto N, Oki M, Nosho K, et al. Frequent epigenetic inactivation of Wnt inhibitory factor-1 in human gastrointestinal cancers. Oncogene. 2005;24:7946-52.

23. Dixon MF, Genta RM, Yardley JH, Correa P. Classification and grading of gastritis. The updated Sydney System. International Workshop on the Histopathology of Gastritis, Houston 1994. Am J Surg Pathol. 1996;20:1161-81.

24. Choi J, Kim SG, Yoon H, Im JP, Kim JS, Kim WH, et al. Eradication of Helicobacter pylori after endoscopic resection of gastric tumors does not reduce incidence of metachronous gastric carcinoma. Clin Gastroenterol Hepatol. 2014;12:793-800, e1.

25. Choi J, Kim SG, Kim BG, Koh SJ, Kim JW, Lee KL. Helicobacter pylori eradication modulates aberrant $\mathrm{CpG}$ island hypermethylation in gastric carcinogenesis. Korean J Gastroenterol. 2016;68:253-9.

26. Park SY, Yoo EJ, Cho NY, Kim N, Kang GH. Comparison of $\mathrm{CpG}$ island hypermethylation and repetitive DNA hypomethylation in premalignant stages of gastric cancer, stratified for Helicobacter pylori infection. J Pathol. 2009;219:410-6.

27. Trinh BN, Long TI, Laird PW. DNA methylation analysis by MethyLight technology. Methods. 2001;25:456-62.

28. Weisenberger DJ, Siegmund KD, Campan M, Young J, Long TI, Faasse MA, et al. CpG island methylator phenotype underlies sporadic microsatellite instability and is tightly associated with BRAF mutation in colorectal cancer. Nat Genet. 2006;38:787-93.

29. Rawson JB, Manno M, Mrkonjic M, Daftary D, Dicks E, Buchanan DD, et al. Promoter methylation of Wnt antagonists DKK1 and SFRP1 is associated with opposing tumor subtypes in two large populations of colorectal cancer patients. Carcinogenesis (Oxf). 2011;32:741-7.

30. Harada T, Yamamoto E, Yamano HO, Nojima M, Maruyama R, Kumegawa $\mathrm{K}$, et al. Analysis of DNA methylation in bowel lavage fluid for detection of colorectal cancer. Cancer Prev Res (Phila). 2014;7:1002-10.

31. Kloten V, Becker B, Winner K, Schrauder MG, Fasching PA, Anzeneder T, et al. Promoter hypermethylation of the tumorsuppressor genes ITIH5, DKK3, and RASSF1A as novel biomarkers for blood-based breast cancer screening. Breast Cancer Res. 2013;15(1):R4.

32. Jiang H, Jin C, Liu J, Hua D, Zhou F, Lou X, et al. Next generation sequencing analysis of miRNAs: MiR-127-3p inhibits glioblastoma proliferation and activates TGF-beta signaling by targeting SKI. OMICS. 2014;18:196-206.

33. Xie Q, Chen L, Shan X, Shan X, Tang J, Zhou F, et al. Epigenetic silencing of SFRP1 and SFRP5 by hepatitis B virus X protein enhances hepatoma cell tumorigenicity through Wnt signaling pathway. Int J Cancer. 2014;135:635-46.

34. Weng LH, Wang CJ, Ko JY, Sun YC, Su YS, Wang FS. Inflammation induction of Dickkopf-1 mediates chondrocyte apoptosis in osteoarthritic joint. Osteoarthr Cartil. 2009; $17: 933-43$.

35. Thompson MC, Fuller C, Hogg TL, Dalton J, Finkelstein D, Lau $\mathrm{CC}$, et al. Genomics identifies medulloblastoma subgroups that are enriched for specific genetic alterations. J Clin Oncol. 2006;24:1924-31.

36. Veeck J, Bektas N, Hartmann A, Kristiansen G, Heindrichs U, Knuchel $\mathrm{R}$, et al. Wnt signalling in human breast cancer: expression of the putative Wnt inhibitor Dickkopf-3 (DKK3) is frequently suppressed by promoter hypermethylation in mammary tumours. Breast Cancer Res. 2008;10:R82.

37. Baker EK, Taylor S, Gupte A, Chalk AM, Bhattacharya S, Green $\mathrm{AC}$, et al. Wnt inhibitory factor 1 (WIF1) is a marker of osteoblastic differentiation stage and is not silenced by DNA methylation in osteosarcoma. Bone (NY). 2015;73:223-32.

38. Livak KJ, Schmittgen TD. Analysis of relative gene expression data using real-time quantitative PCR and the 2(-delta delta $\mathrm{C}(\mathrm{T})$ ) method. Methods. 2001;25:402-8.

39. Yoon H, Kim SG, Kim BK, Shin E, Kim N, Lee HJ, et al. Helicobacter pylori eradication downregulates cellular inhibitor of apoptosis protein 2 in gastric carcinogenesis. Gut Liver. 2017;11:79-86.

40. Romiti A, Zullo A, Borrini F, Sarcina I, Hassan C, Winn S, et al. Relationship between beta-catenin expression and epithelial cell proliferation in gastric mucosa with intestinal metaplasia. World $\mathrm{J}$ Gastroenterol. 2005;11:4400-3.

41. Abe S, Oda I, Suzuki H, Nonaka S, Yoshinaga S, Nakajima T, et al. Long-term surveillance and treatment outcomes of metachronous gastric cancer occurring after curative endoscopic submucosal dissection. Endoscopy. 2015;47:1113-8.

42. Shin CM, Kim N, Jung Y, Park JH, Kang GH, Kim JS, et al. Role of Helicobacter pylori infection in aberrant DNA methylation along multistep gastric carcinogenesis. Cancer Sci. 2010;101:1337-46.

43. Ding SZ, Goldberg JB, Hatakeyama M. Helicobacter pylori infection, oncogenic pathways and epigenetic mechanisms in gastric carcinogenesis. Future Oncol. 2010;6:851-62.

44. Hayashi Y, Tsujii M, Wang J, Kondo J, Akasaka T, Jin Y, et al. CagA mediates epigenetic regulation to attenuate let-7 expression in Helicobacter pylori-related carcinogenesis. Gut. 2013;62:1536-46.

45. Lim JH, Kim SG, Choi J, Im JP, Kim JS, Jung HC. Risk factors for synchronous or metachronous tumor development after endoscopic resection of gastric neoplasms. Gastric Cancer. 2015;18:817-23. 